\title{
Estudio Psicométrico del Inventario para la Planificación de Servicios y la Programación Individual (ICAP), en Población de Estudiantes Chilenos
}

\author{
Psychometric Examination of the Inventory for Client and Agency Planning (ICAP), \\ in Chilean Students
}

\author{
Daniela Vera-Bachmann ${ }^{1}$, José Luis Gálvez-Nieto ${ }^{2}$, Ítalo Trizano-Hermosilla ${ }^{3}$ y Alejandro Álvarez- \\ Espinoza $^{4}$
}

\begin{abstract}
Resumen
El objetivo de este estudio fue analizar las propiedades del ICAP en una población de estudiantes chilenos. Siguiendo las instrucciones originales, el ICAP fue aplicado a una muestra probabilística de padres, madres o cuidadores, de las regiones de Valparaíso, Metropolitana, Libertador Gral. Bernardo O’Higgins, Bío Bío, La Araucanía y Los Lagos, que representaron a un total de 1,670 niños, niñas y adolescentes de ambos sexos (49.3\% hombres y $50.7 \%$ mujeres), con una edad promedio de 10.23 años $(D T=2.95)$. Los resultados evidencian que las saturaciones factoriales de los ítems son claras y permiten definir una estructura adecuada para la medición de la conducta adaptativa, expresada a partir de cuatro dimensiones. Se observaron además, adecuados niveles de fiabilidad para las cuatro dimensiones del constructo. Se concluye que el ICAP entrega evidencias de calidad psicométrica para su utilización en población chilena.
\end{abstract}

Palabras clave: conducta adaptativa, validación de instrumentos, fiabilidad, validez

\begin{abstract}
The objective of this study was to analyze the properties of ICAP in a population of Chilean students. Following the original instructions, ICAP was applied to a probabilistic sample of parents or caregivers, of the Chilean regions of Valparaiso, Metropolitana, Libertador Gral. Bernardo O'Higgins, Bio Bio, La Araucania and Los Lagos, consisted of a total of 1,670 children and adolescents of both sexes $(49.3 \%$ men and $50.7 \%$ women) with a mean age of 10.23 years $(S D=2.95)$. The results of the exploratory and confirmatory factor analysis of ICAP, shows that the factor loadings of the items are clear and adequate levels of reliability was also observed for the four dimensions of the construct. It is concluded that the ICAP presents psychometric qualities and therefore it can be used in Chilean population.
\end{abstract}

Keywords: adaptive behavior, instrument validation, reliability, validity

La presente investigación ha sido financiada por los proyectos FND19-0001 financiado por la Dirección de Investigación de la Universidad de La Frontera, Chile y el proyecto FONIDE N 911407 , denominado "Conducta Adaptativa: explorando instrumentos para su evaluación en estudiantes chilenos", patrocinado por el Fondo de Investigación y Desarrollo en Educación del Ministerio de Educación de Chile.

\footnotetext{
${ }^{1}$ Doctor en Ciencias de la Educación. Magister en Educación. Psicóloga. Académica Universidad Austral de Chile - Sede Puerto Montt, Instituto de Psicología. Los Pinos s/n Balneario Pelluco, Puerto Montt, Chile. Tel.: +56652277153. Email: daniela.vera@uach.cl

${ }^{2}$ Doctor en Ciencias de la Educación. Magister en Gerencia Social. Trabajador Social. Universidad de La Frontera, Académico Departamento de Trabajo Social, Chile. Investigador del Laboratorio de Investigación en Ciencias Sociales Aplicadas (LICSA). Correo: jose.galvez@ufrontera.cl

${ }^{3}$ Doctor y Máster en Metodología de las Ciencias del Comportamiento y de la Salud. Psicólogo. Académico Universidad de La Frontera, Chile. Departamento de Psicología. Correo: italo.trizano@ufrontera.cl

${ }^{4}$ Magíster en Psicología, mención Psicología Educacional. Psicólogo. Académico Universidad Austral de Chile - Sede Puerto Montt, Instituto de Psicología. Los Pinos s/n Balneario Pelluco, Puerto Montt, Chile. Tel.: +56652277153. Correo: aalvarez@spm.uach.cl

Revista Iberoamericana de Diagnóstico y Evaluación - e Avaliação Psicológica. RIDEP · No54 · Vol.1 · 119-129 · 2020

ISSN: 1135-3848 print /2183-6051online
} 


\section{Introducción}

Desde hace décadas, la American Association on Intellectual and Developmental Disabilities (AAIDD) (2010) - ha propuesto tres criterios para el diagnóstico de discapacidad intelectual: la existencia de limitaciones significativas del funcionamiento intelectual, presencia de limitaciones significativas de la conducta adaptativa - concebida genéricamente como las diversas habilidades aprendidas por las personas para funcionar en su vida cotidiana - y una edad de aparición anterior a los 18 años (Montero \& Lagos, 2011). Esta aproximación, implica acercarse a la idea de un diagnóstico de discapacidad intelectual no centrado exclusivamente en medidas de inteligencia (Guillén, Verdugo, Arias, \& Vicente, 2015; Heber, 1959; Rodríguez, 2009; Tassé, 2013; Tassé et al., 2012; Verdugo \& Schalock, 2010). La integralidad que representa tal enfoque es importante, ya que contribuye a compensar algunos de los potenciales efectos discriminadores derivados del uso exclusivo de estas medidas, como la desproporcionada identificación y derivación educativa de niños de minorías étnicas o grupos socioeconómicos desfavorecidos a grupos de educación especial (Coronel, Lacunza, \& Contini de González, 2006; Montero, 2005; Montero \& Lagos, 2011). En este sentido, la inclusión de la conducta adaptativa constituye un avance sustancial y el aspecto central de una noción de discapacidad intelectual que trasciende la dimensión meramente psicométrica (AlarcónLeiva \& Sepúlveda-Dote, 2014; Luckasson et al., 1992; Oakland \& Daley, 2013; Schalock et al., 2010).

Con el fin de contribuir con lo mencionado, en los últimos años se han observado considerables progresos en el desarrollo y validación de escalas destinadas a medir conducta adaptativa (Floyd et al., 2015). Actualmente existirían aproximadamente 200 escalas de conducta adaptativa a nivel internacional (Arias, Verdugo, Navas, \& Gómez, 2013). No obstante, del total de instrumentos disponibles, sólo unos cuantos se basan en la estructura propuesta por la AAIDD para el constructo de conducta adaptativa (habilidades conceptuales, sociales y prácticas) y cuentan con evidencias suficientes de fiabilidad y validez (Schalock et al., 2010; Tassé et al., 2012), se podrian citar como ejemplo: Vineland Adaptive Behavior Scales, segunda edición (Sparrow, Cicchetti, \& Balla, 2005), o Adaptive Behavior Assessment System- II (Harrison \& Oakland, 2003); sin embargo, ninguna de las escalas disponibles se encuentran idioma español.

Por otra parte, tal como plantea Spreat (1999), difícilmente estas escalas pueden ser usadas para diagnosticar, clasificar y diseñar programas de apoyo individualizados de forma simultánea; y además, ninguna de estas medidas están disponibles en español, lo que dificulta su uso en poblaciones como la chilena, aspecto que constituye un desafío con carácter de urgencia.

En Chile, la evaluación de la conducta adaptativa como criterio diagnóstico de estudiantes con déficit intelectual, no es considerada sino hasta fines del año 2009, fecha en que se promulga el Decreto $\mathrm{N}^{\circ} 170$, correspondiente al reglamento de la ley $\mathrm{N}^{\circ} 20.201$. En este Decreto se hace mención - por primera vez de manera formal - a exigencias sobre la evaluación de la conducta adaptativa en personas con discapacidad intelectual (Ministerio de Educación de Chile, 2009a). Esto supone la explícita valoración de la conducta adaptativa como un elemento relevante para el diagnóstico, en concordancia con la postura internacional respecto del mismo tema (Schalock et al., 2010; Tassé, 2013).

Uno de los instrumentos sugeridos por el Ministerio de Educación de Chile para la evaluación de conducta adaptativa es el ICAP Inventario para la Planificación del Servicio y la Programación Individual - (Bruininks, Hill, Weatherman \& Woodcock, 1986; Montero, 1996). Esencialmente, el ICAP es un registro de diagnóstico, datos personales y limitaciones funcionales que presenta la persona en relación a determinadas destrezas básicas para desenvolverse con independencia en su entorno. Se trata de una prueba relativamente sencilla y rápida, que se ha caracterizado por una sólida validez de contenido y de constructo, además de buena validez de criterio (Montero, 1996) y fiabilidad test-retest (Montero, 2005). No obstante, al mismo tiempo que se sugiere el instrumento descrito, se reconoce que en Chile no se han desarrollado estudios psicométricos que permitan avalar la utilización 
Tabla 1. Resultados muestreo

\begin{tabular}{|c|c|c|c|c|c|}
\hline Región & Población & $\%$ Poblacional & Muestra Planificada & $\%$ Muestral & Resultado muestral \\
\hline Valparaíso & 271531 & 13.0 & 155 & 13.0 & 154 \\
\hline Libertador Gral. Bernardo O'Higgins & 148837 & 7.1 & 85 & 7.1 & 93 \\
\hline Biobío & 340532 & 16.3 & 194 & 16.3 & 191 \\
\hline La Araucanía & 166413 & 8.0 & 95 & 8.0 & 316 \\
\hline Los Lagos & 147964 & 7.1 & 84 & 7.1 & 475 \\
\hline Metropolitana de Santiago & 1012546 & 48.5 & 576 & 48.5 & 441 \\
\hline Total & 2087823 & $100 \%$ & 1188 & $100 \%$ & 1670 \\
\hline Dependencia & Población & $\%$ Poblacional & Muestra Planificada & $\%$ Muestral & Resultado muestral \\
\hline Municipal & 783681 & 37.5 & 445 & 41 & 685 \\
\hline Particular subvencionado & 1304142 & 62.5 & 743 & 59 & 985 \\
\hline Total & 2087823 & $100 \%$ & 1188 & $100 \%$ & 1670 \\
\hline
\end{tabular}

Fuente: El total poblacional fue obtenido del Centro de estudios MINEDUC

http://centroestudios.mineduc.cl/tp_modulos/tpm_seccion/contVentana.php?cc=2179

de instrumentos para medir la conducta adaptativa en el contexto nacional (Ministerio de Educación de Chile, 2009b), carencia que produce como consecuencia el que los antecedentes referidos a estas habilidades sean recabados por medio de instrumentos que no han sido adaptados o a través del llamado "juicio clínico" de los/las psicólogos/as (Montero \& Lagos, 2011).

Esto último evidencia una deuda en el ámbito de la atención a la diversidad a la cual este estudio pretende responder, ya que el no contar con instrumentos validados para la población chilena impide que el criterio de la conducta adaptativa sea adecuadamente incorporado a la evaluación, al tiempo que dificulta la práctica de la misma y el establecimiento de apoyos adecuados para los y las estudiantes que lo requieran (Schalock \& Verdugo, 2012; Shogren, Bradley, Gómez, Yeager, \& Schalock, 2011; Thompson et al., 2010; Verdugo, Arias, Gómez, \& Schalock, 2010).

\section{Método}

\section{Participantes}

La selección de los participantes se realizó mediante un muestreo probabilístico estratificado, con un $95.5 \%$ de confianza, un $2.9 \%$ de margen de error y una varianza $p=q=.5$ (Scheaffer, Mendenhall, \& Ott, 1987). Las unidades muestrales fueron centros educativos públicos y particulares subvencionados, de las regiones chilenas de Valparaíso, Metropolitana, Libertador Gral. Bernardo O’Higgins, Bío Bío, La Araucanía y Los Lagos. Siguiendo las instrucciones del instrumento original, éste fue aplicado a padres, madres o cuidadores, que en total representaron un total de 1,670 niños y adolescentes de ambos sexos (49.3\% hombres y $50.7 \%$ mujeres), con un promedio de edad de 10.23 años $(D T=2.95)$. Los resultados del procedimiento de muestreo se presentan a continuación (ver Tabla 1).

\section{Instrumentos}

En concordancia con el propósito del estudio, se trabajó el Inventario para la Planificación de Servicios y la Programación Individual (ICAP), versión española (Montero, 1996).

El ICAP (Bruininks, Hill, Weatherman \& Woodcock, 1986; Montero, 1996) está formado por un registro sistemático de datos sociodemográficos sobre la persona atendida, y además cuenta con dos instrumentos de medición; uno sobre conducta adaptativa y otro sobre problemas conductuales. Este inventario puede ser aplicado a personas de cualquier edad, aunque se orienta hacia personas con algún tipo de discapacidad. El ICAP consta de 77 ítems y evalúa destrezas motoras (DM); destrezas sociales y comunicativas (DSC); así como destrezas para la vida personal (DVP); y destrezas de la vida diaria (DVD). Los ítems de estas dimensiones puntúan en una escala tipo Likert de cuatro categorías $(0=$ Nunca $o$ rara vez, $3=$ La realiza muy bien). Este instrumento mide además, comportamiento autolesivo, hábitos atípicos o estereotipias, retraimiento o falta de atención, agresividad hacia otros, conductas sociales ofensivas, disruptivas y no colaboradoras. En el proceso de adaptación en España, presentó adecuados niveles de confiabilidad los cuales 
oscilaron entre el .86 a .98 en el caso de personas sin discapacidade y entre el .88 y el .98 en el caso de personas con discapacidades (Krause et al., 2016).

\section{Procedimiento}

Para asegurar que las expresiones utilizadas en los ítems fueran comprendidas en Chile, la versión original del instrumento fue sometida a revisión por dos jueces expertos, los cuales tenían conocimiento de la variable en estudio y habían vivido en ambos entornos culturales.

Posteriormente, se desarrolló un focus group con 10 profesionales que se desempeñan en el área de educación especial y que tenían conocimiento previo del Inventario, para asegurar la comprensión de las expresiones utilizadas y las formas de respuestas sugeridas en la versión preliminar. Con las sugerencias, se diagramó la versión final del instrumento.

Finalmente, se tomó contacto con los directores de los establecimientos educativos y se solicitó autorización para acceder a la muestra. En reuniones de padres, se realizó charla informativa y se solicitó la participación voluntaria y anónima a través de la firma de consentimiento informado.

\section{Análisis de datos}

Para responder al propósito del estudio, se tomaron dos muestras independientes. La primera muestra exploratoria $(n=742)$ y la segunda confirmatoria $(n=928)$. Con la primera muestra $(n=742)$ se realizó un Análisis Factorial Exploratorio (AFE) con las regiones de La Araucanía y Los Lagos. Para evaluar la factibilidad de este procedimiento se revisó la adecuación muestral de los datos (Ferrando \& Anguiano-Carrasco, 2010) con el coeficiente Kaiser-Meyer-Olkin (KMO) y el test de esfericidad de Barttlet.

El método de estimación utilizado fue el de Mínimos Cuadrados no Ponderados (ULS) con rotación oblimin $\mathrm{y}$, atendiendo a la naturaleza ordinal de las variables (Flora \& Curran, 2004), se trabajó sobre la matriz de correlaciones policóricas (Asun, Rdz-Navarro, \& Alvarado, 2015) mediante el programa FACTOR versión 10.1 (Lorenzo-Seva \& Ferrado, 2006); software que permite además la imputación de valores perdidos.
Para determinar el número de factores a extraer se utilizó el Método Hull. Respecto a la inclusión de los ítems en cada factor se utilizó como criterio saturaciones mayores o iguales a .4 (Izquierdo, Olea, \& Abad, 2014). De este modo se obtienen dimensiones mejor definidas y con menor redundancia en el contenido de los ítems, mostrando factores más parsimoniosos.

Con la segunda muestra ( $\mathrm{n}=928)$, correspondiente a las regiones de Valparaíso, Libertador General Bernardo O'Higgins, Metropolitana y Bío Bío, se realizó un Análisis Factorial Confirmatorio (AFC) con el software MPLUS 7.11 (Muthén \& Muthén, 2012).

Para la estimación de los índices de bondad de ajuste, se utilizó un método ordinal de estimación (WLSMV) para lo cual se trabajó con correlaciones policóricas. Teniendo en consideración este aspecto, se utilizaron los índices de bondad de ajuste ordinales como ChiCuadrado $\left(\chi^{2}\right)$, índice de ajuste comparativo (CFI), Tucker-Lewis Index (TLI) y Error cuadrático medio de aproximación (RMSEA). Para los primeros los índices CFI y TLI, se consideran como un ajuste adecuado del modelo, valores superiores a .90 (Schumacher \& Lomax, 1996), mientras que para el RMSEA, se considera un ajuste razonable valores inferiores a .08 (Browne \& Cudeck, 1993).

Respecto a las evidencias de fiabilidad del instrumento (Santisteban \& Alvarado, 2001), se trabajó con los coeficientes Alfa ordinal (Gadermann, Guhn, \& Zumbo, 2012), Omega (McDonald, 1999) y el Greatest Lower Bound, (Glb) (Berge \& Sočan, 2004). El coeficiente Alfa de Cronbach (1951) se ha utilizado como la línea de base de las demás estimaciones de la fiabilidad, ya que diversos autores (Cho \& Kim, 2015; Sheng \& Sheng, 2012; TrizanoHermosilla \& Alvarado, 2016) han mostrado que este coeficiente presenta limitaciones al estimar la fiabilidad de los test en ciencias sociales. Para realizar estos análisis se trabajó con el Software R (R Development Core Team, 2013), específicamente con el paquete psych. 
Tabla 2. Medias, desviaciones típicas (DT) y Análisis Factorial Exploratorio

\begin{tabular}{|c|c|c|c|c|c|c|}
\hline \multirow[b]{2}{*}{ Ítems } & \multirow[t]{2}{*}{ Media } & \multirow[t]{2}{*}{$D T$} & \multicolumn{4}{|c|}{ Análisis factorial exploratorio } \\
\hline & & & $\begin{array}{c}\text { Destrezas } \\
\text { de la vida } \\
\text { diaria }\end{array}$ & $\begin{array}{c}\text { Destrezas } \\
\text { motoras }\end{array}$ & $\begin{array}{c}\text { Destrezas } \\
\text { de la vida } \\
\text { personal }\end{array}$ & $\begin{array}{c}\text { Destrezas } \\
\text { sociales y } \\
\text { comunicativas }\end{array}$ \\
\hline 1.- Pasa objetos pequeños de una mano a la otra. & 2.872 & .50 & -.080 & .778 & -.025 & .114 \\
\hline $\begin{array}{l}\text { 2. Se sienta solo/a, manteniendo la cabeza y la espalda derechas y } \\
\text { firmes ( } \sin \text { apoyo) durante treinta segundos. }\end{array}$ & 2.674 & .72 & .003 & .824 & -.042 & .040 \\
\hline $\begin{array}{l}\text { 3.- Se mantiene de pie, al menos durante cinco segundos, } \\
\text { apoyándose en muebles u otros objetos. }\end{array}$ & 2.659 & .83 & .034 & .874 & -.075 & .003 \\
\hline $\begin{array}{l}\text { 4.- Mete objetos pequeños en recipientes y los vuelve a sacar } \\
\text { después. }\end{array}$ & 2.826 & .65 & -.051 & .824 & .161 & -.016 \\
\hline $\begin{array}{l}\text { 5.- Hace rayas, marcas o dibujos, con lápiz o con pinturas, en una } \\
\text { hoja de papel. }\end{array}$ & 2.914 & .45 & -.051 & .582 & .314 & .027 \\
\hline 6.- Escribe su nombre copiándolo de un modelo. & 2.593 & .97 & .045 & .714 & .077 & -.073 \\
\hline $\begin{array}{l}\text { 7.- Levanta y lleva una bolsa llena de objetos por lo menos a una } \\
\text { distancia de seis metros y la deposita en el suelo. }\end{array}$ & 2.807 & .64 & .064 & .696 & -.005 & .208 \\
\hline $\begin{array}{l}\text { 8.- En actividades grupales, espera por lo menos dos minutos a que } \\
\text { le llegue su turno (por ejemplo, espera su turno para patear una } \\
\text { pelota o tomar un trago de agua). }\end{array}$ & 2.671 & .66 & .004 & .241 & .206 & .461 \\
\hline $\begin{array}{l}\text { 9.- Ofrece ayuda a otras personas (ejemplos: mantiene la puerta } \\
\text { abierta para que pase una persona que tiene las manos ocupadas o } \\
\text { recoge un objeto que se le ha caído a alguien). }\end{array}$ & 2.702 & 62 & -.002 & .175 & .064 & .705 \\
\hline $\begin{array}{l}\text { 10.- Se comporta de una manera adecuada, sin llamar la atención } \\
\text { de los demás, cuando está con sus amigos en lugares públicos (por } \\
\text { ejemplo, cine, micro, centro comercial, etc.). }\end{array}$ & 2.584 & .73 & .059 & .235 & .023 & .597 \\
\hline $\begin{array}{l}\text { 11.- Cuenta de manera resumida una historia de forma que otra } \\
\text { persona pueda entenderla (por ejemplo, un programa de TV o una } \\
\text { película de cine). }\end{array}$ & 2.726 & .61 & .107 & .156 & .120 & .495 \\
\hline $\begin{array}{l}\text { 12.- Recuerda o sabe cómo localizar números telefónicos y llama a } \\
\text { sus amigos. }\end{array}$ & 2.584 & .82 & .265 & .031 & .110 & .512 \\
\hline 13.- Traga alimentos blandos. & 2.859 & .51 & .083 & .211 & .443 & .119 \\
\hline 14. Permanece sin orinarse al menos durante tres horas. & 2.822 & .61 & -.007 & .027 & .621 & .179 \\
\hline 15.- Se quita el pantalón o la falda y la ropa interior. & 2.847 & .55 & -.012 & .132 & .754 & .023 \\
\hline $\begin{array}{l}\text { 16.- Hace sus necesidades cuando se le sienta en el WC de acuerdo } \\
\text { a un horario regular o cuando se le lleva al baño. }\end{array}$ & 2.886 & .49 & -.033 & -.017 & .943 & -.005 \\
\hline 17.- Se pone camisetas o polerón, aunque sea al revés. & 2.576 & .96 & .131 & .319 & .468 & -.209 \\
\hline 18.- Lava, seca y luego guarda los platos en su lugar. & 2.072 & 1.13 & .530 & -.142 & .345 & .172 \\
\hline $\begin{array}{l}\text { 19.- Arregla su dormitorio, lo cual incluye guardar su ropa, cambiar } \\
\text { las sábanas, quitar el polvo y barrer el suelo. }\end{array}$ & 1.984 & 1.08 & .556 & -.047 & .136 & .233 \\
\hline $\begin{array}{l}\text { 20.- Prepara listas de compras de por lo menos seis productos para } \\
\text { adquirir en una tienda de comestibles. }\end{array}$ & 1.774 & 1.24 & .659 & .006 & .087 & .138 \\
\hline $\begin{array}{l}\text { 21.- Carga y maneja una lavadora, utilizando la cantidad de } \\
\text { detergente y el programa apropiados. }\end{array}$ & 1.069 & 1.25 & .843 & -.069 & -.018 & .126 \\
\hline $\begin{array}{l}\text { 22.- Planifica, prepara y sirve una comida completa para más de } \\
\text { dos personas. }\end{array}$ & 1.197 & 1.28 & .842 & -.043 & -.018 & .131 \\
\hline $\begin{array}{l}\text { 23.- Realiza pequeñas reparaciones de su ropa, como coserse un } \\
\text { botón, o encarga a la persona adecuada para que lo haga. }\end{array}$ & 1.355 & 1.30 & .773 & -.021 & .072 & .095 \\
\hline $\begin{array}{l}\text { 24.- Maneja herramientas manuales eléctricas, potencialmente } \\
\text { peligrosas, y aparatos de piezas móviles (por ejemplo, un taladro } \\
\text { eléctrico, una batidora o una licuadora). }\end{array}$ & .850 & 1.21 & .909 & .035 & .025 & -.168 \\
\hline $\begin{array}{l}\text { 25.- Administra su dinero de forma que cubra los gastos de, por lo } \\
\text { menos, una semana (ocio, transporte y otras necesidades). }\end{array}$ & 1.597 & 1.25 & .651 & .048 & -.031 & .133 \\
\hline $\begin{array}{l}\text { 26.- Rellena formularios y asiste a entrevistas de selección para } \\
\text { buscar trabajo. }\end{array}$ & .776 & 1.20 & .857 & .063 & -.080 & -.135 \\
\hline $\begin{array}{l}\text { 27.- Efectúa pagos de boletas (agua, luz, internet, etc.) antes de que } \\
\text { venza el plazo. }\end{array}$ & .643 & 1.14 & .894 & .033 & -.097 & -.120 \\
\hline
\end{tabular}

\section{Resultados}

\section{Evidencias de dimensionalidad}

\section{Análisis Factorial Exploratorio}

Los resultados de los coeficientes de adecuación muestral $(\mathrm{KMO}=.899$; Test de Barttlet $(g l=551)=$
$5791,4 ; p<.01)$ indican que la matriz de correlaciones es adecuada para ser factorizada. Luego de realizar análisis factoriales iterativos, considerando el criterio de saturaciones mayores o iguales a .4, se llegó a una solución factorial óptima de 27 ítems en la cual quedan reflejadas cuatro dimensiones teóricas del instrumento, que 
Tabla 3. Medias, desviaciones típicas (DT), Saturaciones del Análisis Factorial Confirmatorio y correlaciones entre escalas

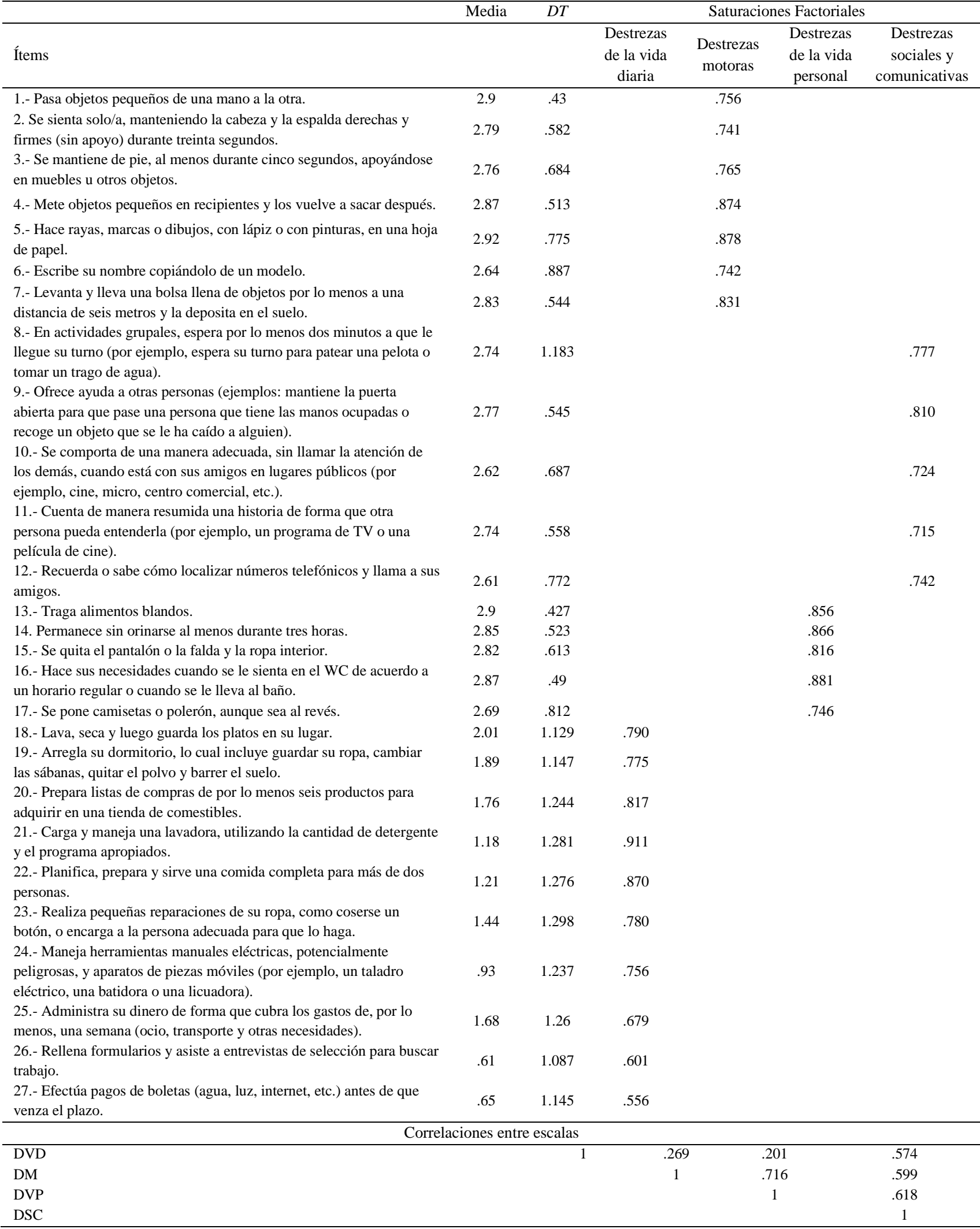

explicaron un $68.3 \%$ de la varianza del constructo. El primer factor denominado "Destrezas de la vida diaria" (DVD) que agrupa 10 ítems (ítems 18 al 27) explicó un 36.8\% de la varianza, el segundo factor agrupa todos los ítems referidos a "Destrezas motoras" (DM) (ítems 1 hasta 7) y explica un $21.5 \%$, el tercer factor denominado "Destrezas de la vida personal" (DVP) que explica 5.2\% (ítems 13 al 17) y el factor "Destrezas Sociales y 
Comunicativas" (DSC) que explica un $4.9 \%$ (ítems 8 al 12).

Como se evidencia en la Tabla 2, las saturaciones factoriales de los ítems son claras y permiten definir una estructura adecuada para la medición de la conducta adaptativa, expresada a partir de cuatro dimensiones, que en términos de estructura teórica resultan comprensibles para las personas evaluadas.

\section{Análisis Factorial Confirmatorio}

Una vez obtenida la solución factorial exploratoria, se realizó un análisis factorial confirmatorio con la segunda muestra (928 participantes), para evaluar el ajuste del modelo empírico de cuatro factores.

Para el modelo propuesto, los índices de bondad de ajuste presentaron los siguientes valores: $\chi^{2}(318)=1102.270, \quad p<.001$; TLI=.933; CFI=.939; RMSEA=.057 (IC90\% .053-.061). Estos valores indican, en general, que el modelo ajusta bien a los datos, por lo que se confirma la estructura empírica propuesta de cuatro factores.

En la Tabla 3 se observan las saturaciones estandarizadas del análisis factorial confirmatorio. Para el primer factor (Destrezas de la Vida Diaria), las saturaciones varían entre .556 y .911 ; para el segundo factor (Destrezas Motoras), las saturaciones van desde .741 hasta .878; por su parte para el tercer factor (Destrezas de la Vida Personal) las saturaciones fluctúan entre .746 y .881 ; finalmente para el factor (Destrezas sociales y comunicativas) las saturaciones variaron entre .715 y .810 . Todas las cargas fueron estadísticamente significativas $(p<.001)$. Además se presentan las correlaciones entre las escalas del ICAP, siendo todas positivas, estadísticamente significativas y de magnitudes moderadas (ver Tabla 3).

\section{Evidencias de fiabilidad}

La fiabilidad por consistencia interna, medida a través del coeficiente Alfa de Cronbach, presenta adecuados valores para las cuatro dimensiones del constructo. Con el objetivo de presentar indicadores adicionales de fiabilidad de la escala, se evaluó también la fiabilidad mediante coeficientes más adecuados para datos ordinales. En la Tabla 4 se presentan los valores de fiabilidad de otros tres coeficientes: Alfa ordinal, Omega y Glb.

Se observa que, en términos generales, los tres coeficientes alternativos tienden a coincidir en sus estimaciones, presentando siempre mejores valores de fiabilidad que el coeficiente Alfa de Cronbach. De este modo, las estimaciones presentadas por estos coeficientes reflejan más adecuadamente la fiabilidad o precisión de las dimensiones que los métodos tradicionales.

De los tres coeficientes es Omega quien presenta, en general, los valores más altos de fiabilidad; por su parte Glb, al ser entendido como el Gran Límite Inferior de la fiabilidad, refleja el valor mínimo teórico que se puede obtener dada una matriz de datos específica, de este modo la verdadera fiabilidad de las dimensiones puede ser cualquier valor comprendido entre el rango de [Glb, 1], por lo que las estimaciones de Alfa de Cronbach resultan sesgadas negativamente al estar fuera de ese rango.

Tabla 4. Fiabilidad de las Dimensiones mediante distintos estimadores

\begin{tabular}{lcccc}
\hline Dimensiones & Alfa & $\begin{array}{c}\text { Alfa } \\
\text { Ordinal }\end{array}$ & Omega & Glb \\
\hline DSC & .626 & .687 & .691 & .700 \\
DM & .802 & .903 & .905 & .878 \\
DVP & .759 & .775 & .792 & .793 \\
DVD & .897 & .928 & .931 & .929 \\
\hline
\end{tabular}

\section{Conclusiones}

El presente estudio tuvo como propósito evaluar las propiedades psicométricas del Inventario para la planificación de servicios y la programación individual (ICAP) en una muestra de estudiantes chilenos, aportando al país un instrumento con adecuadas propiedades psicométricas que avalan su uso - como screening inicial- en la detección de dificultades asociadas a la conducta adaptativa.

Respecto a las evidencias de dimensionalidad del instrumento, es posible señalar que los resultados sugieren la presencia de cuatro factores latentes correlacionados. Los cuatro factores denominados: "Destrezas de la vida diaria", "Destrezas motoras", "Destrezas de la vida 
personal" y "Destrezas sociales y comunicativas", explicaron en conjunto el $68.3 \%$ de la varianza del constructo de habilidades adaptativas en la muestra exploratoria. Esta estructura factorial fue confirmada a través de modelamiento de ecuaciones estructurales para datos ordinales, con la segunda muestra del estudio.

Cabe señalar que el inventario original sufrió una importante disminución de ítems (versión inicial 77 ítems, versión final 27 ítems); sin embargo, a juicio del equipo de investigación constituye una fortaleza, ya que se cuenta con un instrumento más breve, con una estructura factorial sólida, con altas saturaciones factoriales y niveles de precisión adecuados, lo que permite su aplicación de forma más expedita sin perder capacidad explicativa.

En cuanto a la fiabilidad del inventario, los resultados presentan valores adecuados, al igual que lo evidenciado en España por el estudio de Montero (1996). Esto permite fundamentar que la versión de 27 ítems realiza una evaluación precisa del constructo.

Pese a que este estudio responde a algunas de las necesidades reportadas por autores como Montero y Lagos (2011), se sugiere profundizar en las evidencias de validez mediante modelos de invarianza factorial para determinar si el test es equivalente entre personas pertenecientes a distintos grupos, tales como el sexo, tipo de dependencia, pertenencia a una etnia o el nivel socioeconómico.

Por último, es necesario señalar - respecto de las limitaciones del estudio - que, los resultados obtenidos con la versión corta del instrumento son provisionales por lo que es necesario confirmar las propiedades de esta versión abreviada en futuros estudios. Por otra parte, el ICAP es un instrumento orientado a evaluar personas con un grado mayor de discapacidad. Esta característica del instrumento, dificulta la evaluación en personas con grados leves de discapacidad en el ámbito de la conducta adaptativa, como es el caso de los usuarios de los Programas de Integración Escolar (PIE). Esta situación genera que las puntuaciones de las escalas sean en general altas, lo que conlleva a que la gran mayoría de los niños cumplan de manera satisfactoria cada una de los ítems del ICAP, por ejemplo el ítem "Pasa objetos pequeños de una mano a la otra", perteneciente a la dimensión Destrezas Motoras (DM) es logrado en el $97.8 \%$ de la muestra total $(n=1,670)$. Este hallazgo sugiere la necesidad de seguir explorando nuevas escalas para la evaluación de Conducta Adaptativa de manera de continuar perfeccionando el diagnóstico de discapacidad intelectual, reforzando lo que han sugerido previamente autores como Navas, Verdugo, Arias y Gómez (2010).

\section{Referencias}

Alarcón-Leiva, J., \& Sepúlveda-Dote, M. (2014). La conducta adaptativa como criterio diagnóstico de discapacidad intelectual en estudiantes de Chile. Revista Latinoamericana de Ciencias Sociales, Niñez y Juventud, 12(1), 187-199. http://doi.org/10.11600/1692715x.12110091713

American Association on Intellectual and Developmental Disabilities, AAIDD. (2010). Intellectual disability: Definition, classification, and systems of supports (11th Edition). Washington, D.C.: Autor.

Arias, B., Verdugo, M., Navas, P., \& Gómez, L. (2013). Factor structure of the construct of adaptative behavior in children with and withouth intelectual disability. International Journal of Clinical and Health Psychology, 13(2), 133-166.

http://doi.org/10.1016/S1697-2600(13)70019-X

Asún, R. A., Rdz-Navarro, K., \& Alvarado, J. M. (2015). Developing multidimensional Likert scales using item factor analysis: The case of four-point items. Sociological Methods \& Research, 45(1), 109-133. http://doi.org/10.1177/0049124114566716

Berge, J. M. F., \& Sočan, G. (2004). The greatest lower bound to the reliability of a test and the hypothesis of unidimensionality. Psychometrika, 69(4), 613-625. http://doi.org/10.1007/BF02289858

Browne, M., \& Cudeck, R. (1993). Alternative ways of assessing model fit. In: Bollen, K. \& Long, J. (Eds.) Testing Structural Equation Models (pp. 136-162). Beverly Hills, CA: Sage.

Bruininks, R., Hill, B., Weatherman, R., \& Woodcock, R. (1986). ICAP. Inventory for 
Client and Agency Planning. Examiner's Manual. Allen: DLM Teaching Resources.

Cho, E., \& Kim, S. (2015). Cronbach's coefficient alpha: Well known but poorly understood. Organizational Research Methods, 18(2), 207-230. http://doi.org/10.1177/1094428114555994

Coronel,C., Lacunza, A., \& Contini de González, N. (2006). Las habilidades cognitivas en niños privados culturalmente. Resultados preliminares de la primera fase de evaluación. Revista Iberoamericana de Diagnóstico y Evaluación e - Avaliação Psicológica, 2(22), 49-74.

Cronbach, L. (1951). Coefficient alpha and the internal structure of tests. Psychometrika, 16(3), 297-334. http://doi.org/10.1007/BF02310555

Ferrando, P., \& Anguiano-Carrasco, C. (2010). El análisis factorial como técnica de investigación en psicología. Papeles Del Psicólogo, 31(1), 18-33.

Flora, D., \& Curran, P. (2004). An empirical evaluation of alternative methods of estimation for confirmatory factor analysis with ordinal data. Psychological Methods, 9(4), 466-491. http://doi.org/10.1037/1082-989X.9.4.466

Floyd, R., Shands, E., Afonso, V., Phillips, J., Autry, B., Mosteller, J., Skinner, M., \& Irby, S. (2015). A systematic review and psychometric evaluation of Adaptive Behavior Scales and Recommendations for Practice, Journal of Applied School Psychology, 31(1), 83-113. http://doi.org/10.1080/15377903.2014.979384

Gadermann, A. M., Guhn, M., \& Zumbo, B. D. (2012). Estimating ordinal reliability for Likert-type and ordinal item response data: A conceptual, empirical and practical guide. Practical Assessment, Research \& Evaluation, 17(3), 1-13.

Guillén, V. M., Verdugo M. A., Arias, B., \& Vicente, E. (2015). Desarrollo de una escala de evaluación de necesidades de apoyo para niños o adolescentes con discapacidad intelectual. Anales de Psicología, 31(1), 137144.

http://dx.doi.org/10.6018/analesps.31.1.166491
Harrison, P. L., \& Oakland, T. (2003). Adaptive behavior assessment system - Second Edition. San Antonio, TX: Harcourt Assessment.

Heber, R. (1959). A manual on terminology and classification in mental retardation: A monograph supplement. American Journal of Mental Deficiency, 64(2), 1-111. http://doi.org/ 10.1007/BF02172475

Izquierdo, I., Olea, J., \& Abad, F. (2014). Exploratory factor analysis in validation studies: Uses and recommendations. Psicothema, 26(3), 395-400. http://doi.org/10.7334/psicothema2013.349

Krause, A., Román, F., Esarza, Y., Novoa, M., Salinas, P., Toledo, F., \& Vallejos, A. (2016). Caracterización de la conducta adaptativa en escolares con y sin discapacidad intelectual de la región de la Araucanía. Diversitas: Perspectivas en Psicología, 12(1), 27-36. http://dx.doi.org/10.15332/s17949998.2016.0001.02.

Lorenzo-Seva, U., \& Ferrando, P. (2006). FACTOR: A computer program to fit the exploratory factor analysis model. Behavior Research Methods, 38(1), 88-91. doi:10.3758/BF03192753

Luckasson, R., Coulter, D., Polloway, A., Reiss, S., Schalock, R., Snell, E., \& Stark, J. (1992). Mental retardation: Definition, Classification, and systems of supports (9th ed.). Washington: American Association on Mental Retardation.

McDonald, R. (1999). Test theory: A unified treatment. Mahwah, NJ: Lawrence Erlbaum Associates.

Ministerio de Educación de Chile (2009a). Decreto 170. Fija normas para determinar los alumnos con necesidades educativas especiales que serán beneficiarios de las subvenciones para educación especial. Santiago de Chile: Autor.

Ministerio de Educación de Chile (2009b). Orientaciones técnicas para la evaluación diagnóstica de estudiantes que presentan necesidades educativas especiales que serán beneficiarios de subvenciones para educación especial. Santiago de Chile: Autor.

Montero, D. (1996). Evaluación de la Conducta Adaptativa en personas con discapacidades. 
Adaptación y validación del Icap. Bilbao: Mensajero.

Montero, D. (2005). La conducta adaptativa en el panorama científico y profesional actual. Intervención Psicosocial, 14(3), 277-293.

Montero, D., \& Lagos, J. (2011). Conducta adaptativa y discapacidad intelectual: 50 años de historia y su incipiente desarrollo en la educación en Chile. Estudios Pedagógicos, $37(2)$, 345-361. http://dx.doi.org/10.4067/S071807052011000200021

Muthén, L., \& Muthén, B. (2012). Mplus user's guide (6th Ed.). Los Angeles, CA: Muthén \& Muthén.

Navas, P., Verdugo, M. A., Arias, B., \& Gómez, L. (2010). La conducta adaptativa con discapacidad intelectual. Revista española sobre Discapacidad Intelectual, 41(235), 2848.

Oakland, T., \& Daley, M. (2013). Adaptive behavior: Its history, concepts, assessment, and applications. En K. F. Geisinger, B. A. Bracken, J. F. Carlson, J.-I. C. Hansen, N. R. Kuncel, S. P. Reise, \& M. C. Rodríguez (Eds.), APA handbook of testing and assessment in psychology, Vol. 3: Testing and assessment in school psychology and education (pp. 183-212). Washington, DC: American Psychological Association.

R Development Core Team. (2013). R: A Language and Environment for Statistical Computing. ("R Foundation for Statistical Computing," Ed.). Vienna. ISBN: 3-90005107-0.

Rodríguez, M. (2009). Análisis factorial confirmatorio de la versión uruguaya de la escala Smart de Trapnell para medir capacidad intelectual percibida. Revista Iberoamericana de Diagnóstico y Evaluación - e Avaliação Psicológica, 1(27), 85-105.

Santisteban, C., \& Alvarado, J. (2001). Modelos Psicométricos. Madrid: UNED.

Schalock, R., Borthwick-Duffy, S., Bradley, V., Buntinx, W., Coulter, D., Craig, E., ... \& Yeager, M. (2010). Intellectual disability definition, classification and systems of supports (11th Ed). Washington: AAIDD.

Schalock R. L., \& Verdugo M. A. (2012). A leadership guide to redefining intellectual and developmental disabilities organizations: Eight successful change strategies. Baltimore, MD: Brookes.

Scheaffer, R., Mendenhall, W., \& Ott, L. (1987). Elementos de muestreo (G. Rendón \& J. Gómez, Trans.). México D.F., México: Grupo Editorial Iberoamérica.

Sparrow, S., Cicchetti, D., \& Balla, D. (2005). Vineland Adaptive Behavior Scales, Second Edition (Vineland-II). Circle Pines, MN: American Guidance Service.

Sheng, Y., \& Sheng, Z. (2012). Is coefficient alpha robust to non-normal data? Frontiers in Psychology, 3(34), 1-13. http://doi.org/10.3389/fpsyg.2012.00034

Shogren, K. A., Bradley, V. J., Gomez, S. C., Yeager, M. H., \& Schalock, R. L. (2011). Política pública y mejora de los resultados deseados para las personas con discapacidad intelectual. Siglo Cero, 42(2), 7-25.

Schumacher, R. \& Lomax, R. (1996). A beginner's guide to structural equation modeling. New Jersey: Lawrence Erlbaum Associates, Publishers.

Spreat, S. (1999). Psychometric standards for adaptive behavior assessment. En R. Schalock (ed.) Adaptive Behavior and Its Measurement. Implications for the Field of Mental Retardation, (pp. 103-118). Washington, D. C.: AAMR.

Tassé, M. J., Schalock, R. L., Balboni, G., Bersani, H., Duffy, S. A., De Valenzuela, J. S., Spreat, S., Thissen, D. M., Widaman, K. F., \& Zhang, D. (2012). The construct of adaptive behavior: Its conceptualization, measurement, and use in the field of intellectual disability. American Journal on Intellectual and Developmental Disabilities, 117(4), 291-303. https://doi.org/10.1352/1944-7558-117.4.291

Tassé, M. J. (2013). ¿Qué son las limitaciones significativas en conducta adaptativa en personas con discapacidades intelectuales y del desarrollo? Revista española sobre discapacidad intelectual, 44(11), 22-33.

Thompson, J. R., Bradley, V J., Buntinx, W. H. E., Schalock, R. L., Shogren, K. A., Snell Miller, M. E. et al. (2010). Conceptualizando los apoyos y las necesidades de apoyo de 
personas con discapacidad intelectual. Siglo Cero, 41(233), 7-22.

Trizano-Hermosilla, I., \& Alvarado, J. M. (2016). Best alternatives to cronbach's alpha reliability in realistic conditions: Congeneric and asymmetrical measurements. Frontiers in Psychology, 7, 1-8. doi:http://doi.org/10.3389/fpsyg.2016.00769

Verdugo, M. A., Arias, B., Gómez, L. E., \& Schalock, R. L. (2010). Development of an objective instrument to assess quality of life in social services: Reliability and validity in Spain. International Journal of Clinical and Health Psychology, 10(1), 105-123. http://doi.org/10.1111/j.13652788.2012.01585.x

Verdugo, M. A., \& Schalock, R. (2010). Últimos avances en el enfoque y concepción de las personas con discapacidad intelectual. Siglo Cero, 41(4), 7-21. 